\title{
Random homomorphisms and random derivations in random normed algebras via fixed point method
}

\author{
Choonkil Park' ${ }^{1}$ Madjid Eshaghi Gordji ${ }^{2}$ and Reza Saadati ${ }^{3^{*}}$
}

"Correspondence: rsaadati@eml.cc ${ }^{3}$ Department of Mathematics, Iran University of Science and Technology, Tehran, Iran Full list of author information is available at the end of the article

\begin{abstract}
Using the fixed point method, we prove the Hyers-Ulam stability of the Cauchy additive functional inequality and of the Cauchy-Jensen additive functional inequality in random normed spaces.

MSC: $47 \mathrm{H} 10 ; 39 \mathrm{~B} 52 ; 37 \mathrm{H} 10 ; 60 \mathrm{H} 25 ; 17 \mathrm{~B} 40 ; 39 \mathrm{~B} 72 ; 47 \mathrm{~B} 47 ; 54 \mathrm{E} 70$

Keywords: additive functional inequality; fixed point; random derivation; random homomorphism; Hyers-Ulam stability; random normed algebra
\end{abstract}

\section{Introduction and preliminaries}

The stability problem of functional equations originated from the question of Ulam [1] concerning the stability of group homomorphisms. Hyers [2] gave the first affirmative partial answer to the question of Ulam for Banach spaces. Hyers' theorem was generalized by Aoki [3] for additive mappings and by Rassias [4] for linear mappings by considering an unbounded Cauchy difference. The paper of Rassias [4] has provided a lot of influence on the development of what we call Hyers-Ulam stability of functional equations. A generalization of the Rassias theorem was obtained by Găvruta [5] by replacing the unbounded Cauchy difference with a general control function in the spirit of Rassias' approach. Important contributions to Hyers-Ulam stability were made by Forti [6]. For Jensen's functional equation stability, significant generalizations were given by Jung [7] and successively, by Lee and Jun [8] by using the direct method (Hyers-Ulam method).

A Hyers-Ulam stability problem for the quadratic functional equation was proved by Skof [9] for mappings $f: X \rightarrow Y$, where $X$ is a normed space and $Y$ is a Banach space. Cholewa [10] noticed that the theorem of Skof is still true if the relevant domain $X$ is replaced with an Abelian group. The stability problems of several functional equations have been extensively investigated by a number of authors, and there are many interesting results concerning this problem (see [11-27]).

In the sequel, we adopt the usual terminology, notations and conventions of the theory of random normed spaces, as in [28-32]. Throughout this paper, $\Delta^{+}$is the space of distribution functions, that is, the space of all mappings $F: \mathbb{R} \cup\{-\infty, \infty\} \rightarrow[0,1]$ such that $F$ is left-continuous and non-decreasing on $\mathbb{R}, F(0)=0$ and $F(+\infty)=1 . D^{+}$is a subset of $\Delta^{+}$consisting of all functions $F \in \Delta^{+}$for which $l^{-} F(+\infty)=1$, where $l^{-} f(x)$ denotes the left limit of the function $f$ at the point $x$, that is, $l^{-} f(x)=\lim _{t \rightarrow x^{-}} f(t)$. The space $\Delta^{+}$is partially 
ordered by the usual point-wise ordering of functions, i.e., $F \leq G$ if and only if $F(t) \leq G(t)$ for all $t$ in $\mathbb{R}$. The maximal element for $\Delta^{+}$in this order is the distribution function $\varepsilon_{0}$ given by

$$
\varepsilon_{0}(t)= \begin{cases}0, & \text { if } t \leq 0 \\ 1, & \text { if } t>0\end{cases}
$$

Definition $1.1([31])$ A mapping $T:[0,1] \times[0,1] \rightarrow[0,1]$ is a continuous triangular norm (briefly, a continuous $t$-norm) if $T$ satisfies the following conditions:

(a) $T$ is commutative and associative;

(b) $T$ is continuous;

(c) $T(a, 1)=a$ for all $a \in[0,1]$;

(d) $T(a, b) \leq T(c, d)$ whenever $a \leq c$ and $b \leq d$ for all $a, b, c, d \in[0,1]$.

Typical examples of continuous $t$-norms are $T_{P}(a, b)=a b, T_{M}(a, b)=\min (a, b)$ and $T_{L}(a, b)=\max (a+b-1,0)$ (the Lukasiewicz $t$-norm).

Definition 1.2 ([32]) A random normed space (briefly, RN-space) is a triple $(X, \mu, T)$, where $X$ is a vector space, $T$ is a continuous $t$-norm and $\mu$ is a mapping from $X$ into $D^{+}$such that the following conditions hold:

$\left(R N_{1}\right) \mu_{x}(t)=\varepsilon_{0}(t)$ for all $t>0$ if and only if $x=0$;

$\left(R N_{2}\right) \mu_{\alpha x}(t)=\mu_{x}\left(\frac{t}{|\alpha|}\right)$ for all $x \in X, \alpha \neq 0$;

$\left(R N_{3}\right) \mu_{x+y}(t+s) \geq T\left(\mu_{x}(t), \mu_{y}(s)\right)$ for all $x, y \in X$ and all $t, s \geq 0$.

Every normed space $(X,\|\cdot\|)$ defines a random normed space $\left(X, \mu, T_{M}\right)$, where

$$
\mu_{x}(t)=\frac{t}{t+\|x\|}
$$

for all $t>0$, and $T_{M}$ is the minimum $t$-norm. This space is called the induced random normed space.

Definition 1.3 A random normed algebra is a random normed space with algebraic structure such that $\left(R N_{4}\right) \mu_{x y}(t s) \geq \mu_{x}(t) \mu_{y}(s)$ for all $x, y \in X$ and all $t, s>0$.

Example 1.4 Every normed algebra $(X,\|\cdot\|)$ defines a random normed algebra $\left(X, \mu, T_{M}\right)$, where

$$
\mu_{x}(t)=\frac{t}{t+\|x\|}
$$

for all $t>0$. This space is called the induced random normed algebra.

\section{Definition 1.5}

(1) Let $\left(X, \mu, T_{M}\right)$ and $\left(Y, \mu, T_{M}\right)$ be random normed algebras. An $\mathbb{R}$-linear mapping $f: X \rightarrow Y$ is called a random homomorphism if $f(x y)=f(x) f(y)$ for all $x, y \in X$.

(2) An $\mathbb{R}$-linear mapping $f: X \rightarrow X$ is called a random derivation if $f(x y)=f(x) y+x f(y)$ for all $x, y \in X$. 
Definition 1.6 Let $(X, \mu, T)$ be an RN-space.

(1) A sequence $\left\{x_{n}\right\}$ in $X$ is said to be convergent to $x$ in $X$ if, for every $\epsilon>0$ and $\lambda>0$, there exists a positive integer $N$ such that $\mu_{x_{n}-x}(\epsilon)>1-\lambda$ whenever $n \geq N$.

(2) A sequence $\left\{x_{n}\right\}$ in $X$ is called a Cauchy sequence if, for every $\epsilon>0$ and $\lambda>0$, there exists a positive integer $N$ such that $\mu_{x_{n}-x_{m}}(\epsilon)>1-\lambda$ whenever $n \geq m \geq N$.

(3) An RN-space $(X, \mu, T)$ is said to be complete if and only if every Cauchy sequence in $X$ is convergent to a point in $X$.

Theorem 1.7 ([31]) If $(X, \mu, T)$ is an $R N$-space and $\left\{x_{n}\right\}$ is a sequence such that $x_{n} \rightarrow x$, then $\lim _{n \rightarrow \infty} \mu_{x_{n}}(t)=\mu_{x}(t)$ almost everywhere.

Let $X$ be a set. A function $d: X \times X \rightarrow[0, \infty]$ is called a generalized metric on $X$ if $d$ satisfies the following:

(1) $d(x, y)=0$ if and only if $x=y$;

(2) $d(x, y)=d(y, x)$ for all $x, y \in X$;

(3) $d(x, z) \leq d(x, y)+d(y, z)$ for all $x, y, z \in X$.

We recall a fundamental result in fixed point theory.

Theorem 1.8 ([33-35]) Let $(X, d)$ be a complete generalized metric space and let $J: X \rightarrow X$ be a strictly contractive mapping with the Lipschitz constant $L<1$. Then for each given element $x \in X$, either

$$
d\left(J^{n} x, J^{n+1} x\right)=\infty
$$

for all nonnegative integers $n$ or there exists a positive integer $n_{0}$ such that

(1) $d\left(J^{n} x, J^{n+1} x\right)<\infty, \forall n \geq n_{0}$;

(2) the sequence $\left\{J^{n} x\right\}$ converges to a fixed point $y^{*}$ of $J$;

(3) $y^{\prime \prime}$ is the unique fixed point of $J$ in the set $Y=\left\{y \in X \mid d\left(J^{n_{0}} x, y\right)<\infty\right\}$;

(4) $d\left(y, y^{*}\right) \leq \frac{1}{1-L} d(y, J y)$ for all $y \in Y$.

In 1996, Isac and Rassias [36] were the first to provide applications of the stability theory of functional equations for the proof of new fixed point theorems with applications. Starting with 2003, the fixed point alternative was applied to investigate the Hyers-Ulam stability for Jensen's functional equation in $[26,33,37]$ as well as for the Cauchy functional equation in [38] (see also [39] for quadratic functional equations, [40] for monomial functional equations and [41] for operatorial equations etc.). By using fixed point methods, the stability problems of several functional equations have been extensively investigated by a number of authors (see [26, 29, 33, 37-40, 42-44]).

Gilányi [45] showed that if $f$ satisfies the functional inequality

$$
\|2 f(x)+2 f(y)-f(x-y)\| \leq\|f(x+y)\|,
$$

then $f$ satisfies the Jordan-von Neumann functional equation

$$
2 f(x)+2 f(y)=f(x+y)+f(x-y) .
$$


See also [46]. Fechner [47] and Gilányi [48] proved the Hyers-Ulam stability of the functional inequality (1.1). Park, Cho and Han [49] investigated the Cauchy additive functional inequality

$$
\|f(x)+f(y)+f(z)\| \leq\|f(x+y+z)\|
$$

and the Cauchy-Jensen additive functional inequality

$$
\|f(x)+f(y)+f(2 z)\| \leq\left\|2 f\left(\frac{x+y}{2}+z\right)\right\|
$$

and proved the Hyers-Ulam stability of the functional inequalities (1.2) and (1.3) in Banach spaces.

Throughout this paper, assume that $\left(X, \mu, T_{M}\right)$ is a random normed algebra and that $\left(Y, \mu, T_{M}\right)$ is a complete random normed algebra.

The Hyers-Ulam stability of different functional equations in random normed and fuzzy normed spaces has been recently studied in [29, 30, 39, 50-53]. They are completed with the recent paper [54], which contains some stability results for functional equations in probabilistic metric and random normed spaces.

This paper is organized as follows. In Section 2, we prove the Hyers-Ulam stability of random homomorphisms in complete random normed algebras associated with the Cauchy additive functional inequality (1.2). In Section 3, we prove the Hyers-Ulam stability of random derivations in complete random normed algebras associated with the Cauchy-Jensen additive functional inequality (1.3).

\section{Stability of random homomorphisms in random normed algebras}

In this section, using the fixed point method, we prove the Hyers-Ulam stability of random homomorphisms in complete random normed algebras associated with the Cauchy additive functional inequality (1.2).

Theorem 2.1 Let $\varphi: X^{3} \rightarrow[0, \infty)$ be a function such that there exists an $L<\frac{1}{2}$ with

$$
\varphi(x, y, z) \leq \frac{L}{2} \varphi(2 x, 2 y, 2 z)
$$

for all $x, y, z \in X$. Let $f: X \rightarrow Y$ be an odd mapping satisfying

$$
\begin{aligned}
& \mu_{r f(x)+f(r y)+f(r z)}(t) \geq \min \left\{\mu_{f(r x+r y+r z)}\left(\frac{t}{2}\right), \frac{t}{t+\varphi(x, y, z)}\right\}, \\
& \mu_{f(x y)-f(x) f(y)}(t) \geq \frac{t}{t+\varphi(x, y, 0)}
\end{aligned}
$$

for all $r \in \mathbb{R}$, all $x, y, z \in X$ and all $t>0$. Then $H(x):=\lim _{n \rightarrow \infty} 2^{n} f\left(\frac{x}{2^{n}}\right)$ exists for each $x \in X$ and defines a random homomorphism $H: X \rightarrow Y$ such that

$$
\mu_{f(x)-A(x)}(t) \geq \frac{(2-2 L) t}{(2-2 L) t+L \varphi(x, x,-2 x)}
$$


Proof Since $f$ is odd, $f(0)=0$. So $\mu_{f(0)}\left(\frac{t}{2}\right)=1$. Letting $r=1$ and $y=x$ and replacing $z$ by $-2 x$ in (2.1), we get

$$
\mu_{f(2 x)-2 f(x)}(t) \geq \frac{t}{t+\varphi(x, x,-2 x)}
$$

for all $x \in X$.

Consider the set

$$
S:=\{g: X \rightarrow Y\}
$$

and introduce the generalized metric on $S$ :

$$
d(g, h)=\inf \left\{v \in \mathbb{R}_{+}: \mu_{g(x)-h(x)}(v t) \geq \frac{t}{t+\varphi(x, x,-2 x)}, \forall x \in X, \forall t>0\right\},
$$

where, as usual, $\inf \phi=+\infty$. It is easy to show that $(S, d)$ is complete (see the proof of [30, Lemma 2.1]).

Now we consider the linear mapping $J: S \rightarrow S$ such that

$$
J g(x):=2 g\left(\frac{x}{2}\right)
$$

for all $x \in X$.

Let $g, h \in S$ be given such that $d(g, h)=\varepsilon$. Then

$$
\mu_{g(x)-h(x)}(\varepsilon t) \geq \frac{t}{t+\varphi(x, x,-2 x)}
$$

for all $x \in X$ and all $t>0$. Hence

$$
\begin{aligned}
\mu_{g g(x)-J h(x)}(L \varepsilon t) & =\mu_{2 g\left(\frac{x}{2}\right)-2 h\left(\frac{x}{2}\right)}(L \varepsilon t) \\
& =\mu_{g\left(\frac{x}{2}\right)-h\left(\frac{x}{2}\right)}\left(\frac{L}{2} \varepsilon t\right) \\
& \geq \frac{\frac{L t}{2}}{\frac{L t}{2}+\varphi\left(\frac{x}{2}, \frac{x}{2},-x\right)} \geq \frac{\frac{L t}{2}}{\frac{L t}{2}+\frac{L}{2} \varphi(x, x,-2 x)} \\
& =\frac{t}{t+\varphi(x, x,-2 x)}
\end{aligned}
$$

for all $x \in X$ and all $t>0$. So $d(g, h)=\varepsilon$ implies that $d(J g, J h) \leq L \varepsilon$. This means that

$$
d(J g, J h) \leq L d(g, h)
$$

for all $g, h \in S$.

It follows from (2.4) that

$$
\mu_{f(x)-2 f\left(\frac{x}{2}\right)}\left(\frac{L}{2} t\right) \geq \frac{t}{t+\varphi(x, x,-2 x)}
$$

for all $x \in X$ and all $t>0$. So $d(f, J f) \leq \frac{L}{2}$. 
By Theorem 1.8, there exists a mapping $H: X \rightarrow Y$ satisfying the following:

(1) $H$ is a fixed point of $J$, i.e.,

$$
H\left(\frac{x}{2}\right)=\frac{1}{2} H(x)
$$

for all $x \in X$. Since $f: X \rightarrow Y$ is odd, $H: X \rightarrow Y$ is an odd mapping. The mapping $H$ is a unique fixed point of $J$ in the set

$$
M=\{g \in S: d(f, g)<\infty\}
$$

This implies that $H$ is a unique mapping satisfying (2.5) such that there exists a $v \in(0, \infty)$ satisfying

$$
\mu_{f(x)-H(x)}(v t) \geq \frac{t}{t+\varphi(x, x,-2 x)}
$$

for all $x \in X$;

(2) $d\left(J^{n} f, H\right) \rightarrow 0$ as $n \rightarrow \infty$. This implies the equality

$$
\lim _{n \rightarrow \infty} 2^{n} f\left(\frac{x}{2^{n}}\right)=H(x)
$$

for all $x \in X$;

(3) $d(f, H) \leq \frac{1}{1-L} d(f, J f)$, which implies the inequality

$$
d(f, H) \leq \frac{L}{2-2 L} .
$$

This implies that the inequality (2.3) holds.

Let $r=1$ in (2.1). By (2.1),

$$
\begin{aligned}
& \mu_{2^{n}\left(f\left(\frac{x}{2^{n}}\right)+f\left(\frac{y}{2^{n}}\right)+f\left(\frac{-x-y}{2^{n}}\right)\right)}\left(2^{n} t\right) \\
& \geq \min \left\{\mu_{2^{n} f(0)}\left(2^{n-1} t\right), \frac{t}{t+\varphi\left(\frac{x}{2^{n}}, \frac{y}{2^{n}}, \frac{-x-y}{2^{n}}\right)}\right\}=\frac{t}{t+\varphi\left(\frac{x}{2^{n}}, \frac{y}{2^{n}}, \frac{-x-y}{2^{n}}\right)}
\end{aligned}
$$

for all $x, y \in X$, all $t>0$ and all $n \in \mathbb{N}$. So

$$
\mu_{2^{n}\left(f\left(\frac{x}{2^{n}}\right)+f\left(\frac{y}{2^{n}}\right)+f\left(\frac{-x-y}{2^{n}}\right)\right)}(t) \geq \frac{\frac{t}{2^{n}}}{\frac{t}{2^{n}}+\frac{L^{n}}{2^{n}} \varphi(x, y,-x-y)}
$$

for all $x, y \in X$, all $t>0$ and all $n \in \mathbb{N}$. Since $\lim _{n \rightarrow \infty} \frac{\frac{t}{2^{n}}}{\frac{t}{2^{n}} \frac{L^{n}}{2^{n}} \varphi(x, y,-x-y)}=1$ for all $x, y \in X$ and all $t>0$,

$$
\mu_{H(x)+H(y)+H(-x-y)}(t) \geq 1
$$

for all $x, y \in X$ and all $t>0$. So the mapping $H: X \rightarrow Y$ is Cauchy additive. 
Let $y=-x$ and $z=0$ in (2.1). By (2.1),

$$
\mu_{2^{n} f\left(\frac{r x}{2^{n}}\right)-2^{n} r f\left(\frac{x}{2^{n}}\right)}\left(2^{n} t\right) \geq \frac{t}{t+\varphi\left(\frac{x}{2^{n}}, \frac{-x}{2^{n}}, 0\right)}
$$

for all $r \in \mathbb{R}$, all $x \in X$, all $t>0$ and all $n \in \mathbb{N}$. So

$$
\mu_{2^{n} f\left(\frac{r x}{2^{n}}\right)-2^{n} r f\left(\frac{x}{2^{n}}\right)}(t) \geq \frac{\frac{t}{2^{n}}}{\frac{t}{2^{n}}+\frac{L^{n}}{2^{n}} \varphi(x,-x, 0)}
$$

for all $r \in \mathbb{R}$, all $x \in X$, all $t>0$ and all $n \in \mathbb{N}$. Since $\lim _{n \rightarrow \infty} \frac{\frac{t}{2^{n}}}{\frac{t}{2^{n}}+\frac{L^{n}}{2^{n}} \varphi(x,-x, 0)}=1$ for all $x \in X$ and all $t>0$,

$$
\mu_{H(r x)-r H(x)}(t)=1
$$

for all $r \in \mathbb{R}$, all $x \in X$ and all $t>0$. Thus the additive mapping $H: X \rightarrow Y$ is $\mathbb{R}$-linear.

By (2.2),

$$
\mu_{4^{n} f\left(\frac{x}{2^{n}} \cdot \frac{y}{2^{n}}\right)-2^{n} f\left(\frac{x}{2^{n}}\right) \cdot 2^{n} f\left(\frac{y}{2^{n}}\right)}\left(4^{n} t\right) \geq \frac{t}{t+\varphi\left(\frac{x}{2^{n}}, \frac{y}{2^{n}}, 0\right)}
$$

for all $x, y \in X$, all $t>0$ and all $n \in \mathbb{N}$. So

$$
\mu_{4^{n} f\left(\frac{x}{2^{n}} \cdot \frac{y}{2^{n}}\right)-2^{n} f\left(\frac{x}{2^{n}}\right) \cdot 2^{n} f\left(\frac{y}{2^{n}}\right)}(t) \geq \frac{\frac{t}{4^{n}}}{\frac{t}{4^{n}}+\frac{L^{n}}{2^{n}} \varphi(x, y, 0)}
$$

for all $x, y \in X$, all $t>0$ and all $n \in \mathbb{N}$. Since $\lim _{n \rightarrow \infty} \frac{\frac{t}{4^{n}}}{\frac{t}{4^{n}}+\frac{L^{n}}{2^{n}} \varphi(x, y, 0)}=1$ for all $x, y \in X$ and all $t>0$,

$$
\mu_{H(x y)-H(x) H(y)}(t)=1
$$

for all $x, y \in X$ and all $t>0$. Thus the mapping $H: X \rightarrow Y$ is multiplicative.

Therefore, there exists a unique random homomorphism $H: X \rightarrow Y$ satisfying (2.3).

Theorem 2.2 Let $\varphi: X^{3} \rightarrow[0, \infty)$ be a function such that there exists an $L<1$ with

$$
\varphi(x, y, z) \leq 2 L \varphi\left(\frac{x}{2}, \frac{y}{2}, \frac{z}{2}\right)
$$

for all $x, y, z \in X$. Let $f: X \rightarrow Y$ be an odd mapping satisfying (2.1) and (2.2). Then $H(x):=$ $\lim _{n \rightarrow \infty} \frac{1}{2^{n}} f\left(2^{n} x\right)$ exists for each $x \in X$ and defines a random homomorphism $H: X \rightarrow Y$ such that

$$
\mu_{f(x)-H(x)}(t) \geq \frac{(2-2 L) t}{(2-2 L) t+\varphi(x, x,-2 x)}
$$

for all $x \in X$ and all $t>0$. 
Proof Let $(S, d)$ be the generalized metric space defined in the proof of Theorem 2.1.

Consider the linear mapping $J: S \rightarrow S$ such that

$$
J(x):=\frac{1}{2} g(2 x)
$$

for all $x \in X$.

It follows from (2.4) that

$$
\mu_{f(x)-\frac{1}{2} f(2 x)}\left(\frac{1}{2} t\right) \geq \frac{t}{t+\varphi(x, x,-2 x)}
$$

for all $x \in X$ and all $t>0$. So $d(f, J f) \leq \frac{1}{2}$.

By Theorem 1.8, there exists a mapping $H: X \rightarrow Y$ satisfying the following:

(1) $H$ is a fixed point of $J$, i.e.,

$$
H(2 x)=2 H(x)
$$

for all $x \in X$. Since $f: X \rightarrow Y$ is odd, $H: X \rightarrow Y$ is an odd mapping. The mapping $H$ is a unique fixed point of $J$ in the set

$$
M=\{g \in S: d(f, g)<\infty\}
$$

This implies that $H$ is a unique mapping satisfying (2.7) such that there exists a $v \in(0, \infty)$ satisfying

$$
\mu_{f(x)-H(x)}(v t) \geq \frac{t}{t+\varphi(x, x,-2 x)}
$$

for all $x \in X$;

(2) $d\left(J^{n} f, H\right) \rightarrow 0$ as $n \rightarrow \infty$. This implies the equality

$$
\lim _{n \rightarrow \infty} \frac{1}{2^{n}} f\left(2^{n} x\right)=H(x)
$$

for all $x \in X$;

(3) $d(f, H) \leq \frac{1}{1-L} d(f, J f)$, which implies the inequality

$$
d(f, H) \leq \frac{1}{2-2 L}
$$

This implies that the inequality (2.6) holds.

The rest of the proof is similar to the proof of Theorem 2.1.

\section{Stability of random derivations on random normed algebras}

In this section, using the fixed point method, we prove the Hyers-Ulam stability of random derivations on complete random normed algebras associated with the Cauchy-Jensen additive functional inequality (1.3). 
Theorem 3.1 Let $\varphi: Y^{3} \rightarrow[0, \infty)$ be a function such that there exists an $L<\frac{1}{2}$ with

$$
\varphi(x, y, z) \leq \frac{L}{2} \varphi(2 x, 2 y, 2 z)
$$

for all $x, y, z \in Y$. Let $f: Y \rightarrow Y$ be an odd mapping satisfying

$$
\begin{aligned}
& \mu_{r f(x)+f(r y)+r f(2 z)}(t) \geq \min \left\{\mu_{2 f\left(\frac{r x+r y}{2}+r z\right)}\left(\frac{2 t}{3}\right), \frac{t}{t+\varphi(x, y, z)}\right\}, \\
& \mu_{f(x y)-f(x) y-x f(y)}(t) \geq \frac{t}{t+\varphi(x, y, 0)}
\end{aligned}
$$

for all $r \in \mathbb{R}$, all $x, y, z \in Y$ and all $t>0$. Then $D(x):=\lim _{n \rightarrow \infty} 2^{n} f\left(\frac{x}{2^{n}}\right)$ exists for each $x \in Y$ and defines a random derivation $D: Y \rightarrow Y$ such that

$$
\mu_{f(x)-D(x)}(t) \geq \frac{(2-2 L) t}{(2-2 L) t+L \varphi(x, x,-x)}
$$

for all $x \in Y$ and all $t>0$.

Note that $\mu_{f(0)}\left(\frac{2 t}{3}\right)=1$.

Proof Letting $y=x=-z$ in (3.1), we get

$$
\mu_{f(2 x)-2 f(x)}(t) \geq \frac{t}{t+\varphi(x, x,-x)}
$$

for all $x \in Y$.

Consider the set

$$
S:=\{g: Y \rightarrow Y\}
$$

and introduce the generalized metric on $S$ :

$$
d(g, h)=\inf \left\{v \in \mathbb{R}_{+}: \mu_{g(x)-h(x)}(v t) \geq \frac{t}{t+\varphi(x, x,-x)}, \forall x \in Y, \forall t>0\right\},
$$

where, as usual, $\inf \phi=+\infty$. It is easy to show that $(S, d)$ is complete (see the proof of [30, Lemma 2.1]).

Now we consider the linear mapping $J: S \rightarrow S$ such that

$$
J g(x):=2 g\left(\frac{x}{2}\right)
$$

for all $x \in Y$.

It follows from (3.4) that

$$
\mu_{f(x)-2 f\left(\frac{x}{2}\right)}\left(\frac{L}{2} t\right) \geq \frac{t}{t+\varphi(x, x,-x)}
$$

for all $x \in Y$ and all $t>0$. So $d(f, J f) \leq \frac{L}{2}$. 
By Theorem 1.8, there exists a mapping $D: Y \rightarrow Y$ satisfying the following:

(1) $D$ is a fixed point of $J$, i.e.,

$$
D\left(\frac{x}{2}\right)=\frac{1}{2} D(x)
$$

for all $x \in Y$. Since $f: Y \rightarrow Y$ is odd, $D: Y \rightarrow Y$ is an odd mapping. The mapping $D$ is a unique fixed point of $J$ in the set

$$
M=\{g \in S: d(f, g)<\infty\}
$$

This implies that $D$ is a unique mapping satisfying (3.5) such that there exists a $v \in(0, \infty)$ satisfying

$$
\mu_{f(x)-D(x)}(v t) \geq \frac{t}{t+\varphi(x, x,-x)}
$$

for all $x \in Y$;

(2) $d\left(J^{n} f, D\right) \rightarrow 0$ as $n \rightarrow \infty$. This implies the equality

$$
\lim _{n \rightarrow \infty} 2^{n} f\left(\frac{x}{2^{n}}\right)=D(x)
$$

for all $x \in Y$;

(3) $d(f, D) \leq \frac{1}{1-L} d(f, J f)$, which implies the inequality

$$
d(f, D) \leq \frac{L}{2-2 L}
$$

This implies that the inequality (3.3) holds.

Let $r=1$ in (3.1). By (3.1),

$$
\begin{aligned}
& \mu_{2^{n}\left(f\left(\frac{x}{2^{n}}\right)+f\left(\frac{y}{2^{n}}\right)+f\left(\frac{-x-y}{2^{n}}\right)\right)}\left(2^{n} t\right) \\
& \geq \min \left\{\mu_{2^{n+1} f(0)}\left(\frac{2^{n} t}{3}\right), \frac{t}{t+\varphi\left(\frac{x}{2^{n}}, \frac{y}{2^{n}}, \frac{-x-y}{2^{n+1}}\right)}\right\}=\frac{t}{t+\varphi\left(\frac{x}{2^{n}}, \frac{y}{2^{n}}, \frac{-x-y}{2^{n+1}}\right)}
\end{aligned}
$$

for all $x, y \in Y$, all $t>0$ and all $n \in \mathbb{N}$. So

$$
\mu_{2^{n}\left(f\left(\frac{x}{2^{n}}\right)+f\left(\frac{y}{2^{n}}\right)+f\left(\frac{-x-y}{2^{n}}\right)\right)}(t) \geq \frac{\frac{t}{2^{n}}}{\frac{t}{2^{n}}+\frac{L^{n}}{2^{n}} \varphi\left(x, y, \frac{-x-y}{2}\right)}
$$

for all $x, y \in Y$, all $t>0$ and all $n \in \mathbb{N}$. Since $\lim _{n \rightarrow \infty} \frac{\frac{t}{2^{n}}}{\frac{t}{2^{n}}+\frac{L^{n}}{2^{n}} \varphi\left(x, y, \frac{-x-y}{2}\right)}=1$ for all $x, y \in Y$ and all $t>0$,

$$
\mu_{D(x)+D(y)+D(-x-y)}(t) \geq 1
$$

for all $x, y \in Y$ and all $t>0$. So the mapping $D: Y \rightarrow Y$ is Cauchy additive. 
Let $r=1, z=0$ and $y=-x$ in (3.1). By (3.1),

$$
\mu_{2^{n} f\left(\frac{r x}{2^{n}}\right)-2^{n} r f\left(\frac{x}{2^{n}}\right)}\left(2^{n} t\right) \geq \frac{t}{t+\varphi\left(\frac{x}{2^{n}}, \frac{-x}{2^{n}}, 0\right)}
$$

for all $r \in \mathbb{R}$, all $x \in Y$, all $t>0$ and all $n \in \mathbb{N}$. So

$$
\mu_{2^{n} f\left(\frac{r x}{2^{n}}\right)-2^{n} r f\left(\frac{x}{2^{n}}\right)}(t) \geq \frac{\frac{t}{2^{n}}}{\frac{t}{2^{n}}+\frac{L^{n}}{2^{n}} \varphi(x,-x, 0)}
$$

for all $r \in \mathbb{R}$, all $x \in Y$, all $t>0$ and all $n \in \mathbb{N}$. Since $\lim _{n \rightarrow \infty} \frac{\frac{t}{2^{n}}}{\frac{t}{2^{n}}+\frac{L^{n}}{2^{n}} \varphi(x,-x, 0)}=1$ for all $x \in Y$ and all $t>0$,

$$
\mu_{D(r x)-r D(x)}(t)=1
$$

for all $r \in \mathbb{R}$, all $x \in Y$ and all $t>0$. Thus the additive mapping $D: Y \rightarrow Y$ is $\mathbb{R}$-linear.

By (3.2),

$$
\mu_{4^{n} f\left(\frac{x}{2^{n}} \cdot \frac{y}{2^{n}}\right)-2^{n} f\left(\frac{x}{2^{n}}\right) \cdot y-x \cdot 2^{n} f\left(\frac{y}{2^{n}}\right)}\left(4^{n} t\right) \geq \frac{t}{t+\varphi\left(\frac{x}{2^{n}}, \frac{y}{2^{n}}, 0\right)}
$$

for all $x, y \in Y$, all $t>0$ and all $n \in \mathbb{N}$. So

$$
\mu_{4^{n} f\left(\frac{x}{2^{n}} \cdot \frac{y}{2^{n}}\right)-2^{n} f\left(\frac{x}{2^{n}}\right) \cdot y-x \cdot 2^{n} f\left(\frac{y}{2^{n}}\right)}(t) \geq \frac{\frac{t}{4^{n}}}{\frac{t}{4^{n}}+\frac{L^{n}}{2^{n}} \varphi(x, y, 0)}
$$

for all $x, y \in Y$, all $t>0$ and all $n \in \mathbb{N}$. Since $\lim _{n \rightarrow \infty} \frac{\frac{t}{4^{n}}}{\frac{t}{4^{n}}+\frac{L^{n}}{2^{n}} \varphi(x, y)}=1$ for all $x, y \in Y$ and all $t>0$,

$$
\mu_{D(x y)-D(x) y-x D(y)}(t)=1
$$

for all $x, y \in Y$ and all $t>0$. Thus the mapping $D: Y \rightarrow Y$ satisfies $D(x y)=D(x) y+x D(y)$ for all $x, y \in Y$.

Therefore, there exists a unique random derivation $D: Y \rightarrow Y$ satisfying (3.3).

Theorem 3.2 Let $\varphi: Y^{3} \rightarrow[0, \infty)$ be a function such that there exists an $L<1$ with

$$
\varphi(x, y, z) \leq 2 L \varphi\left(\frac{x}{2}, \frac{y}{2}, \frac{z}{2}\right)
$$

for all $x, y, z \in Y$. Let $f: Y \rightarrow Y$ be an odd mapping satisfying (3.1) and (3.2). Then $D(x):=$ $\lim _{n \rightarrow \infty} \frac{1}{2^{n}} f\left(2^{n} x\right)$ exists for each $x \in Y$ and defines a random derivation $D: Y \rightarrow Y$ such that

$$
\mu_{f(x)-D(x)}(t) \geq \frac{(2-2 L) t}{(2-2 L) t+\varphi(x, x,-x)}
$$

for all $x \in Y$ and all $t>0$. 
Proof Let $(S, d)$ be the generalized metric space defined in the proof of Theorem 3.1. Consider the linear mapping $J: S \rightarrow S$ such that

$$
J g(x):=\frac{1}{2} g(2 x)
$$

for all $x \in Y$.

The rest of the proof is similar to the proofs of Theorems 2.1 and 3.1.

\section{Competing interests}

The authors declare that they have no competing interests.

\section{Authors' contributions}

All authors conceived of the study, participated in its design and coordination, drafted the manuscript, participated in the sequence alignment, and read and approved the final manuscript.

\section{Author details}

${ }^{1}$ Department of Mathematics, Research Institute for Natural Sciences, Hanyang University, Seoul, 133-791, Korea.

${ }^{2}$ Department of Mathematics, Semnan University, P.O. Box 35195-363, Semnan, Iran. ${ }^{3}$ Department of Mathematics, Iran University of Science and Technology, Tehran, Iran.

Received: 7 March 2012 Accepted: 20 August 2012 Published: 4 September 2012

\section{References}

1. Ulam, SM: A Collection of the Mathematical Problems. Interscience, New York (1960)

2. Hyers, DH: On the stability of the linear functional equation. Proc. Natl. Acad. Sci. USA 27, $222-224$ (1941)

3. Aoki, T: On the stability of the linear transformation in Banach spaces. J. Math. Soc. Jpn. 2, 64-66 (1950)

4. Rassias, TM: On the stability of the linear mapping in Banach spaces. Proc. Am. Math. Soc. 72, 297-300 (1978)

5. Găvruta, P: A generalization of the Hyers-Ulam-Rassias stability of approximately additive mappings. J. Math. Anal. Appl. 184, 431-436 (1994)

6. Forti, GL: An existence and stability theorem for a class of functional equations. Stochastica 4, 22-30 (1980)

7. Jung, S: Hyers-Ulam-Rassias stability of Jensen's equation and its application. Proc. Am. Math. Soc. 126, 3137-3143 (1998)

8. Lee, Y, Jun, K: A generalization of the Hyers-Ulam-Rassias stability of Jensen's equation. J. Math. Anal. Appl. 238, 305-315 (1999)

9. Skof, F: Proprietà locali e approssimazione di operatori. Rend. Semin. Mat. Fis. Milano 53, 113-129 (1983)

10. Cholewa, PW: Remarks on the stability of functional equations. Aequ. Math. 27, 76-86 (1984)

11. Aczel, J, Dhombres, J: Functional Equations in Several Variables. Cambridge University Press, Cambridge (1989)

12. Bavand Savadkouhi, M, Eshaghi Gordji, M, Rassias, JM, Ghobadipour, N: Approximate ternary Jordan derivations on Banach ternary algebras. J. Math. Phys. 50, Article ID 042303 (2009)

13. Bourgin, DG: Classes of transformations and bordering transformations. Bull. Am. Math. Soc. 57, 223-237 (1951)

14. Chung, JK, Sahoo, PK: On the general solution of a quartic functional equation. Bull. Korean Math. Soc. 40, 565-576 (2003)

15. Czerwik, S: On the stability of the quadratic mapping in normed spaces. Abh. Math. Semin. Univ. Hamb. 62, 59-64 (1992)

16. Ebadian, A, Ghobadipour, N, Gordji, ME: A fixed point method for perturbation of bimultipliers and Jordan bimultipliers in $C^{*}$-ternary algebras. J. Math. Phys. 51, Article ID 103508 (2010)

17. Gajda, Z: On stability of additive mappings. Int. J. Math. Math. Sci. 14, 431-434 (1991)

18. Hyers, DH, Isac, G, Rassias, TM: Stability of Functional Equations in Several Variables. Birkhäuser, Basel (1998)

19. Jung, S: Hyers-Ulam-Rassias Stability of Functional Equations in Mathematical Analysis. Hadronic Press, Palm Harbor (2001)

20. Kim, $\mathrm{H}$ : On the stability problem for a mixed type of quartic and quadratic functional equation. J. Math. Anal. Appl. 324, 358-372 (2006)

21. Park, C, Eshaghi Gordji, M: Comment on 'Approximate ternary Jordan derivations on Banach ternary algebras [Bavand Savadkouhi et al., J. Math. Phys. 50 (2009), Article ID 042303]'. J. Math. Phys. 51, Article ID 044102 (2010)

22. Rassias, JM, Kim, H: Approximate homomorphisms and derivations between $C^{*}$-ternary algebras. J. Math. Phys. 49 Article ID 063507 (2008)

23. Rassias, TM: On the stability of functional equations and a problem of Ulam. Acta Appl. Math. 62, 23-130 (2000)

24. Rassias, TM, Shibata, K: Variational problem of some quadratic functionals in complex analysis. J. Math. Anal. Appl. 228, 234-253 (1998)

25. Kannappan, P: Quadratic functional equation and inner product spaces. Results Math. 27, 368-372 (1995)

26. Radu, V: The fixed point alternative and the stability of functional equations. Fixed Point Theory 4, 91-96 (2003)

27. Rassias, TM, Šemrl, P: On the Hyers-Ulam stability of linear mappings. J. Math. Anal. Appl. 173, 325-338 (1993)

28. Chang, SS, Cho, YJ, Kang, SM: Nonlinear Operator Theory in Probabilistic Metric Spaces. Nova Science Publishers, New York (2001)

29. Mihet, D: The fixed point method for fuzzy stability of the Jensen functional equation. Fuzzy Sets Syst. 160 1663-1667 (2009) 
30. Miheț, D, Radu, V: On the stability of the additive Cauchy functional equation in random normed spaces. J. Math. Anal. Appl. 343, 567-572 (2008)

31. Schweizer, B, Sklar, A: Probabilistic Metric Spaces. North-Holland, New York (1983)

32. Sherstnev, AN: On the notion of a random normed space. Dokl. Akad. Nauk SSSR 149, 280-283 (1963) (in Russian)

33. Cădariu, L, Radu, V: Fixed points and the stability of Jensen's functional equation. J. Inequal. Pure Appl. Math. 4, Article ID 4 (2003)

34. Diaz, J, Margolis, B: A fixed point theorem of the alternative for contractions on a generalized complete metric space. Bull. Am. Math. Soc. 74, 305-309 (1968)

35. Rus, IA: Principles and Applications of Fixed Point Theory. Dacia, Cluj-Napoca (1979) (in Romanian)

36. Isac, G, Rassias, TM: Stability of $\psi$-additive mappings: applications to nonlinear analysis. Int. J. Math. Math. Sci. 19, 219-228 (1996)

37. Cădariu, L, Radu, V: The fixed point method to stability properties of a functional equation of Jensen type. An. Stiint. Univ. Al. I. Cuza lasi, Mat. 54, 307-318 (2008)

38. Cădariu, L, Radu, V: On the stability of the Cauchy functional equation: a fixed point approach. Grazer Math. Ber. 346, 43-52 (2004)

39. Mirzavaziri, M, Moslehian, MS: A fixed point approach to stability of a quadratic equation. Bull. Braz. Math. Soc. 37 , 361-376 (2006)

40. Cădariu, L, Radu, V: Remarks on the stability of monomial functional equations. Fixed Point Theory 8, 201-218 (2007)

41. Rus, IA: Remarks on Ulam stability of the operatorial equations. Fixed Point Theory 10, 305-320 (2009)

42. Cădariu, L, Radu, V: Fixed point methods for the generalized stability of functional equations in a single variable. Fixed Point Theory Appl. 2008, Article ID 749392 (2008)

43. Park, C: Fixed points and Hyers-Ulam-Rassias stability of Cauchy-Jensen functional equations in Banach algebras. Fixed Point Theory Appl. 2007, Article ID 50175 (2007)

44. Park, C: Generalized Hyers-Ulam-Rassias stability of quadratic functional equations: a fixed point approach. Fixed Point Theory Appl. 2008, Article ID 493751 (2008)

45. Gilányi, A: Eine zur Parallelogrammgleichung äquivalente Ungleichung. Aequ. Math. 62, 303-309 (2001)

46. Rätz, J: On inequalities associated with the Jordan-von Neumann functional equation. Aequ. Math. 66, 191-200 (2003)

47. Fechner, W: Stability of a functional inequalities associated with the Jordan-von Neumann functional equation. Aequ. Math. 71, 149-161 (2006)

48. Gilányi, A: On a problem by K. Nikodem. Math. Inequal. Appl. 5, 707-710 (2002)

49. Park, C, Cho, Y, Han, M: Functional inequalities associated with Jordan-von Neumann type additive functional equations. J. Inequal. Appl. 2007, Article ID 41820 (2007)

50. Miheț, D: The probabilistic stability for a functional equation in a single variable. Acta Math. Hung. 123, 249-256 (2009)

51. Mirmostafaee, AK, Mirzavaziri, M, Moslehian, MS: Fuzzy stability of the Jensen functional equation. Fuzzy Sets Syst. 159, 730-738 (2008)

52. Mirmostafaee, AK, Moslehian, MS: Fuzzy versions of Hyers-Ulam-Rassias theorem. Fuzzy Sets Syst. 159, $720-729$ (2008)

53. Mirmostafaee, AK, Moslehian, MS: Fuzzy approximately cubic mappings. Inf. Sci. 178, 3791-3798 (2008)

54. Cădariu, L, Radu, V: Fixed points and stability for functional equations in probabilistic metric and random normed spaces. Fixed Point Theory Appl. 2009, Article ID 589143 (2009)

doi:10.1186/1029-242X-2012-194

Cite this article as: Park et al.: Random homomorphisms and random derivations in random normed algebras via fixed point method. Journal of Inequalities and Applications 2012 2012:194.

\section{Submit your manuscript to a SpringerOpen ${ }^{\circ}$ journal and benefit from:}

- Convenient online submission

Rigorous peer review

- Immediate publication on acceptance

- Open access: articles freely available online

- High visibility within the field

- Retaining the copyright to your article 